\title{
Wavelet Filtering in Shock Stochastic Systems with High Availability
}

\author{
Sinitsyn Igor Nikolaevich*, Sinitsyn Vladimir Igorevich, Korepanov Edward Rudolfovich, \\ Konashenkova Tatyana Dmitirievna
}

Federal Research Centre "Computer Sciences and Control”, Moscow, Russia

Email address:

sinitsin@dol.ru (S. I. Nikolaevich)

${ }^{*}$ Corresponding author

\section{To cite this article:}

Sinitsyn Igor Nikolaevich, Sinitsyn Vladimir Igorevich, Korepanov Edward Rudolfovich, Konashenkova Tatyana Dmitirievna. Wavelet Filtering in Shock Stochastic Systems with High Availability. International Journal of Systems Engineering. Vol. 5, No. 1, 2021 , pp. 1-12. doi: $10.11648 /$ j.ijse.20210501.11

Received: January 19, 2021; Accepted: February 2, 2021; Published: February 23, 2021

\begin{abstract}
For filtering problems in StSHA under nonGaussian ShD methodological and algorithmically WL support is developed. 3 types of filters are considered: KBF (WLKBF), LPF (WLLPF) and SOLF (WLSOLF). These filters have the following advantages: on-line regime, high accuracy and possibility of algorithmically description of complex ShD. Wavelet filter modifications are based on Galerkin method and Haar wavelet expansions. WLF unlike KBF, LPF and SOLF do not need to integrate system of ordinary differential Eqs. These filters must solve system of linear algebraic Eqs with constant coefficients. KBF (WLKBF) and SOLF (WLSOLF) are recommended for StSHA with additive ShD whereas LPF (WLLPF) are recommended for StSHA with parametric and additive ShD. Basic applications are: on-line identification and calibration of nonstationary processes in StSHA of ShD. Methods are illustrated by example of 3 dimensional differential linear information control system at complex ShD. Basic algorithms and error analysis for KBF (WLKBF) and LPF (WLLPF) are presented and 15 Figure; illustrate filters peculiarities for small and fin damping. These filters allow to estimate the accumulation effects for systematic and random errors. Results may be generalized for filtration, extrapolation with interpolation problems in StSHA and multiple ShD.
\end{abstract}

Keywords: Kalman-Bucy WLF, Linear Pugachev WLF, Shock Disturbances, Stochastic Systems with High Availability (StSHA), Suboptimal Linear WLF, Wavelet Filtering (WLF)

\section{Introduction}

In series [1-6] methodological support for on-line express analysis of stochastic systems with high availability (StSHA) functioning at shock disturbances (ShD) was presented. Special attention was paid to wavelet methods and software tools. Wavelet modifications of Kalan-Bucy filters (WLKBF) for nonstationary linear StSHA at complex ShD were given and illustrated. Wavelet modifications of linear mean square (m.s.) conditionally optimal (Pugachev) filter (WLLPF) for StSHA with parametric ShD are presented and illustrated. Comparative computer results were described. Instrumental accuracy of WLKBF and WLLPF was considered.

Let generalize [1] for KBF, LPF and suboptimal linearized filters (SOLF) in case of non Gaussian ShD. Section 2 is dedicated to KBF and WLKBF. LPF and WLLPF are described in Section 3. In Section 4 SOLF based on linearization by known exact shock distributions are considered. Basic Propositions 1-5 are illustrated by 3 dimensional information control system at deterministic and stochastic ShD.

\section{Kalman-Bucy Filters at Shock Disturbances}

Kalman-Bucy Filter (KBF) for linear nonstationary StSHA is widely used for on-line analysis and synthesis problems. $\mathrm{KBF}$ is based on the following proposition [7-11].

Proposition 1. Let nonstationary differential StSHA being described by the following Eqs: 


$$
\begin{gathered}
\dot{X}_{t}=a_{0 t}^{s h}+a_{1}^{s h} X_{t}+V_{1}^{s h}, \\
Z_{t}=\dot{Y}_{t}=b_{1} X_{t}+V_{2} .
\end{gathered}
$$

Here $\dot{X}_{t}, Y_{t}$ are states and observation vectors, $V_{1}^{s h}=V_{1}$ and $V_{2}$ are independent white noises (in strict sense) and nonGaussian in general case with intensity matrices $v_{1}^{s h}=v_{1}$ and $v_{2}$. Then at nonsingular observation noise $\left(\left|\operatorname{det} v_{2}\right| \neq 0\right)$ $\mathrm{KBF}$ equations are as follows:

$$
\begin{gathered}
\dot{\hat{X}}_{t}=a_{0 t}^{s h}+a_{1}^{s h} \hat{X}_{t}+\beta_{t}\left(Z_{t}-b_{1} \hat{X}_{t}\right), \quad \hat{X}\left(t_{0}\right)=\hat{X}_{0}, \\
\beta_{t}=R_{t} b_{1}^{T} v_{2}^{-1}, \\
\dot{R}_{t}=a_{1} R_{t}+R_{t} a_{1}^{T}+v_{1 t}^{s h}-\beta_{t} v_{2} \beta_{t}^{T}, \quad R\left(t_{0}\right)=R_{0},
\end{gathered}
$$

where $\hat{X}_{t}$ being mean square error estimate of $X_{t} ; R_{t}$ being error covariance matrix; $\beta_{t}$ being matrix amplifier.

Remark. 1. Calculation of $R_{t}$ and $\beta_{t}$ does not need current observation and may be calculated a priori.

For getting Eqs for WLKBF let us exchange variable according to the following Eqs:

$$
\begin{gathered}
\bar{t}=\frac{t-t_{0}}{T-t_{0}}, \quad \bar{t} \in[0,1], \\
\hat{\bar{X}}(\bar{t})=\hat{X}\left(\left(T-t_{0}\right) \bar{t}+t_{0}\right), \quad \hat{\bar{X}}(0)=\hat{X}_{0}, \\
\bar{A}(\bar{t})=\left(T-t_{0}\right)\left[a_{0 t}^{s h}\left(\left(T-t_{0}\right) \bar{t}+t_{0}\right)+\right. \\
\left.+\beta_{t}\left(\left(T-t_{0}\right) \bar{t}+t_{0}\right) Z_{t}\left(\left(T-t_{0}\right) \bar{t}+t_{0}\right)\right], \quad \hat{\bar{X}}(0)=\hat{X}_{0}, \\
\bar{A}(\bar{t})=\left(T-t_{0}\right)\left[a_{1}-\beta_{t}\left(\left(T-t_{0}\right) \bar{t}+t_{0}\right) b_{1}\right] .
\end{gathered}
$$

As a result, we have

$$
\hat{\bar{X}}^{\prime}(\bar{t})=\bar{A}_{0 \bar{t}}+\bar{A}_{\bar{t}} \hat{\bar{X}}(\bar{t}), \quad \hat{\bar{X}}(0)=\hat{X}_{0} .
$$

Remark. 2. Further we put $t=\bar{t}$.

Following [10-14] we introduce Haar wavelets $w_{i}$ and integral of $w_{i}$ using formulae

$$
\begin{gathered}
w_{1}(t)=\varphi(t)=\varphi_{00}(t)=\left\{\begin{array}{lll}
1 & \text { at } & t \in[0,1), \\
0 & \text { at } & t \notin[0,1),
\end{array}\right. \\
w_{2}(t)=\psi(t)=\psi_{00}(t)=\left\{\begin{array}{ccc}
1 & \text { at } & t \in[0,1 / 2), \\
-1 & \text { at } & t \in[1 / 2,1), \\
0 & \text { at } & t \notin[0,1),
\end{array}\right. \\
w_{i}(t)=\psi_{j k}(t)=\left\{\begin{array}{ccc}
\sqrt{2^{j}} & \text { at } & t \in[k / l,(k+0.5) / l), \\
-\sqrt{2^{j}} & \text { at } & t \in[(k+0.5) / l,(k+1) / l), \\
0 & \text { at } & t \notin[k / l,(k+1) / l) .
\end{array}\right.
\end{gathered}
$$

Here $\varphi=\varphi(t)$ being scale function; $\psi=\psi(t)$ mother wavelet

$$
\psi_{j k}=\psi_{j k}(t)=\sqrt{2^{j}} \psi\left(2^{j} t-k\right)
$$

$$
k=0,1, \ldots, l-1 ; l=2^{j} ; j=1,2, \ldots, J ; \quad L=2 \times 2^{j},
$$
$i=l+k+1 ; \quad i=3,4, \ldots, L ; \quad J$ being maximal level of wavelet resolution.

Define integrals $p_{i}$ by formulae

$$
p_{i}(t)=\int_{0}^{t} w_{i}(t) d t \quad(i=1,2, \ldots, L),
$$

where

$$
p_{1}(t)=\left\{\begin{array}{lll}
t & \text { at } & t \in[0,1), \\
0 & \text { at } & t \notin[0,1),
\end{array} \quad p_{i}(t)=\left\{\begin{array}{c}
\sqrt{2^{j}} t-(k / l) \text { at } t \in[k / l,(k+0.5) / l), \\
-\sqrt{2^{j}} t-((k+1) / l)-t \text { at } t \in[(k+0.5) / l,(k+1) / l), \\
0 \text { at } t \notin[k / l,(k+1) / l)
\end{array} \quad(i=2,3, \ldots, L)\right.\right.
$$

For every component $\hat{\bar{X}}_{h} \quad(h=1,2, \ldots, p)$ for $\hat{\bar{X}}(\bar{t})$ Eq (10) gives the following expression:

$$
\hat{\bar{X}}_{h}^{\prime}(\bar{t})=\bar{A}_{h 0}+\sum_{k=1}^{p} \bar{A}_{h k} \hat{\bar{X}}_{k}
$$

Let us expand the drerivative $\hat{\bar{X}}_{h}^{\prime}$ into Haar series:

$$
\hat{\bar{X}}_{h}^{\prime}(\bar{t})=\sum_{i=1}^{L} c_{h i} w_{i}
$$

$$
c_{h i}(\bar{t})=\int_{0}^{1} \hat{\bar{X}}_{h}^{\prime} w_{i} d \tau .
$$

Then we get for Eq (16) solution

$$
\hat{\bar{X}}_{h}(\bar{t})=\sum_{i=1}^{L} c_{h i} p_{i}+\hat{X}_{0 h} .
$$

After substitution Eq (17) and Eq (19) into Eq (16) we have the following expression for $c_{h i}$ : 


$$
\begin{array}{r}
\sum_{i=1}^{L} c_{h i} w_{i}=\bar{A}_{h 0}+\sum_{k=1}^{p} \bar{A}_{h k}\left(\sum_{i=1}^{L} c_{h i} p_{i}+\hat{X}_{0 h} \cdot\right) \quad(20) \quad \begin{array}{c}
\text { After projecting Eq }(20) \\
\text { consideration } w_{i} \text { orthonorm } \\
\text { dimensional system of linear }
\end{array} \\
c_{h s}=\sum_{k=1}^{p} \sum_{i=1}^{L} c_{h i}\left(\bar{A}_{h k} p_{i}, w_{s}\right)+\hat{X}_{0 h} \sum_{i=1}^{L}\left(\bar{A}_{h k}, w_{s}\right)+\left(\bar{A}_{h 0}, w_{s}\right) \quad(s=1,2, \ldots, L) .
\end{array}
$$

After projecting $\mathrm{Eq}$ (20) on basis $w_{i}$, taking into consideration $w_{i}$ orthonormality we come to $(L \times p)$ dimensional system of linear algebraic equations (SLAEq):

Putting

$$
\begin{gathered}
\bar{A}_{h k} p_{i}=\sum_{j=1}^{L} g_{j}^{h k i} w_{j}, \quad \bar{A}_{h k}=\sum_{j=1}^{L} q_{j}^{h k} w_{j}, \quad \bar{A}_{h 0}=\sum_{j=1}^{L} \rho_{j}^{h} w_{j}, \\
g_{j}^{h k i}=\left(\bar{A}_{h k} p_{i}, w_{j}\right)=\int_{0}^{1} \bar{A}_{h k} p_{i} w_{j} d \tau \\
q_{j}^{h k}=\left(\bar{A}_{h k}, w_{j}\right)=\int_{0}^{1} \bar{A}_{h k} w_{j} d \tau \\
\rho_{j}^{h}=\left(\bar{A}_{h 0}, w_{j}\right)=\int_{0}^{1} \bar{A}_{h} w_{j} d \tau
\end{gathered}
$$

we rewrite Eqs (22) in the final form:

$$
c_{h s}=\sum_{k=1}^{p} \sum_{i=1}^{L} c_{h i} g_{s}^{h k i}+\hat{X}_{0 h} \sum_{i=1}^{L} q_{s}^{h k}+\rho_{s}^{h} \quad(s=1,2, \ldots, L) .
$$

Thus we have Proposition 2 [6].

Proposition 2. At conditions

(i) scalar functions $\hat{\bar{X}}_{h}^{\prime}, \hat{\bar{X}}_{h}, \bar{A}_{h k}, \bar{A}_{h 0} \quad(h, k=1,2, \ldots, p)$ belong to space $L^{2}[0,1]$,

(ii) Haar functions $w_{i}, p_{i}$ are defined in space $L^{2}[0,1]$.

Then Eqs for WLKBF being (19) at conditions (24).

Note that Proposition 2 is the basis of corresponding algorithm for calculating m.s. estimation of state StSHA vector described by Eqs (1), (2):

1) $R_{t}$ and $\beta_{t}$ off-line calculation according to Eqs (4), (5).

2) Off-line definition of Haar $w_{i}, p_{i}$ in space $L^{2}[0,1]$ according to Eqs (11)-(14) with maximal level of wavelet resolution $J$.

3) Off-line calculation of $p_{i}$ according to Eq (15).

4) Off-line reduction of Eq (3) to Eq (10).

5) Assign values of observation $Z(t) \quad\left(t \in\left[t_{0}, T\right]\right)$ at points $t_{j}=t_{0}(j-1) \Delta t$ for $j=1,2, \ldots, L ; L=2 \cdot 2^{j} ; \Delta t=\left(T-t_{0}\right) /(L-1)$.

6) On-line composition and solution SLAEq (24) for coefficients $c_{h s}$.

7) On-line calculation of m.s. estimate $\hat{\bar{X}}_{h}$ for every component $\bar{X}_{t}$.

8) On-line transition from $\bar{t} \in[1,0], t \in[1,0]$ and calculation of m.s. estimate $\hat{X}(t)$ according to formula $\hat{\bar{X}}(\bar{t})=\hat{X}\left(\left(T-t_{0}\right) \bar{t}+t_{0}\right)$.

\section{Linear Pugachev Filter at Shock Disturbances}

Let us consider the following StSHA described by Eqs with shock parametric noises:

$$
\begin{aligned}
& \dot{X}_{t}=a_{0 t}+a_{1 t} X_{t}+a_{t} Y_{t}+\left(c_{10 t}+\sum_{r=1}^{n_{Y}} c_{1 r t} Y_{r}+\sum_{r=1}^{n_{X}} c_{1, n_{y}+r, t} X_{r}\right) V, \quad X\left(t_{0}\right)=X_{0}, \\
& \dot{Y}_{t}=b_{0 t}+b_{t} Y_{t}+b_{1 t} X_{t}+\left(c_{20 t}+\sum_{r=1}^{n_{Y}} c_{2 r t} Y_{r}+\sum_{r=1}^{n_{X}} c_{2, n_{y}+r, t} X_{r}\right) V, \quad Y\left(t_{0}\right)=Y_{0},
\end{aligned}
$$

where $V$ being vector white noise. In this case LPF is defined by the following proposition [6].

Proposition 3. Let StSHA at ShD is describe by Eqs:

$$
\dot{\hat{X}}_{t}=a_{0 t}+a_{1 t} \hat{X}_{t}+a_{t} Y_{t}+\beta_{t}\left[Z_{t}-\left(b_{0 t}+b_{t} Y_{t}+b_{1 t} \hat{X}_{t}\right)\right], \quad \hat{X}\left(t_{0}\right)=\hat{X}_{0} .
$$

Probabilistic moments of first and second order of $\left[Y_{1} \ldots Y_{n_{Y}} X_{1} \ldots X_{n_{X}}\right]^{T}$ satisfy the following Eqs:

$$
\begin{gathered}
\dot{m}_{t}=a_{0 t}+a_{1 t} m_{t}, \quad m_{t_{0}}=m_{0}, \\
\dot{K}_{t}=\bar{a}_{t} K_{t}+K_{t} \bar{a}_{t}^{T}+\bar{c}_{0 t} v_{t} \bar{c}_{0 t}^{T}+\sum_{r=1}^{n_{Y}+n_{X}}\left(\bar{c}_{0 t} v_{t} \bar{c}_{r t}^{T}+\bar{c}_{r t} v_{t} \bar{c}_{0 t}^{T}\right) m_{r t}+\sum_{r, s=1}^{n_{Y}+n_{X}} \bar{c}_{0 t} v_{t} \bar{c}_{s t}^{T}\left(m_{r t} m_{s t}+K_{r s}\right), \quad K_{t_{0}}=K_{0},
\end{gathered}
$$


where

$$
\bar{a}_{t}=\left[\begin{array}{ll}
b_{t} & b_{1 t} \\
a_{t} & a_{1 t}
\end{array}\right], \quad \bar{a}_{0 t}=\left[\begin{array}{c}
b_{0 t} \\
a_{0 t}
\end{array}\right], \quad \bar{c}_{r t}=\left[\begin{array}{c}
c_{2 t t} \\
c_{1 r t}
\end{array}\right],
$$

Error covariance matrix $R_{t}$ satisfy Eq

$$
\begin{aligned}
& \dot{R}_{t}=a_{t} R_{t}+R_{t} a_{1 t}^{T}-\left[R_{t} b_{1 t}^{T}+\left(c_{10 t}+\sum_{r=1}^{n_{Y}+n_{X}} c_{1 r t} m_{r t}\right) \times v_{t}\left(c_{20 t}^{T}+\sum_{r=1}^{n_{Y}+n_{X}} c_{2 r t}^{T} m_{r t}\right)+\sum_{r, s=1}^{n_{Y}+n_{X}} c_{1 r t} v_{t} c_{2 s t}^{T} K_{r s}\right] \times \\
& \times \mathfrak{x}_{11 t}^{-1}\left[R_{t} b_{1 t}+\left(c_{20 t}+\sum_{r=1}^{n_{Y}+n_{X}} c_{2 r t} m_{r t}\right) v_{t}\left(c_{10 t}^{T}+\sum_{r=1}^{n_{Y}+n_{X}} c_{1 r t}^{T} m_{r t}\right)+\sum_{r, s=1}^{n_{Y}+n_{X}} c_{2 r t} v_{t} K_{r s}\right]+\left(c_{10 t}+\sum_{r=1}^{n_{Y}+n_{X}} c_{1 r t} m_{r t}\right) \\
& \times v_{t}\left(c_{20 t}^{T}+\sum_{r=1}^{n_{Y}+n_{X}} c_{1 r t}^{T} m_{r t}\right)+\sum_{r, s=1}^{n_{Y}+n_{X}} c_{1 r t} v_{t} c_{1 s t}^{T} K_{r s},
\end{aligned}
$$

where $\mathfrak{x}_{11 t}$ and $\beta_{t}$ as follows

$$
\begin{gathered}
æ_{11 t}=\left(c_{20 t}+\sum_{r=1}^{n_{Y}+n_{X}} c_{2 r t} m_{r t}\right) \times v_{t}\left(c_{20 t}^{T}+\sum_{r=1}^{n_{Y}+n_{X}} c_{2 r t}^{T} m_{r t}\right)+\sum_{r, s=1}^{n_{Y}+n_{X}} c_{2 r t} v_{t} c_{2 s t}^{T} K_{r s}, \\
\beta_{t}=\left[R_{t} b_{1 t}^{T}+\left(c_{10 t}+\sum_{r=1}^{n_{Y}+n_{X}} c_{1 r t} m_{r t}\right) \times v_{t}\left(c_{20 t}^{T}+\sum_{r=1}^{n_{Y}+n_{X}} c_{2 r t}^{T} m_{r t}\right)+\sum_{r, s=1}^{n_{Y}+n_{X}} c_{1 r t} v_{t} c_{2 s t}^{T} K_{r s}\right] \mathfrak{x}_{11 t}^{-1} .
\end{gathered}
$$

Remark. 3. LPF as KBF does not depend on current observations and the basic calculations may be performed a priori. For WLLPF we have the following Eqs [6]:

$$
\begin{gathered}
\left.\hat{\bar{X}}^{\prime}(\bar{t})=\tilde{A}_{0 \bar{t}}+\tilde{A}_{t} \hat{\bar{X}}(\bar{t}), \quad \hat{\bar{X}}^{(\bar{t}}\right)=\hat{X}_{0}(\bar{t}), \\
\hat{\bar{X}}_{h}^{\prime}=\tilde{A}_{h 0}+\sum_{k=1}^{n_{X}} \tilde{A}_{h k} \hat{\bar{X}}_{k}, \\
\hat{\bar{X}}_{h}=\sum_{i=1}^{L} \tilde{c}_{h i} p_{i}+\hat{X}_{o h}, \\
\tilde{c}_{h s}=\sum_{k=1}^{p} \sum_{i=1}^{L} \tilde{c}_{h i} \tilde{g}_{s}^{h k i}+\hat{X}_{o h} \sum_{i=1}^{L} \tilde{q}_{s}^{h k}+\tilde{\rho}_{s}^{h}\left(h=1,2, \ldots, n_{X}, s=1,2, \ldots, L\right), \\
\tilde{A}_{h k} p_{i}=\sum_{j=1}^{L} \tilde{g}_{j}^{h k i} w_{j}, \quad \tilde{A}_{h k}=\sum_{j=1}^{L} \tilde{q}_{j}^{h k} w_{j}, \quad \tilde{A}_{0 h}=\sum_{j=1}^{L} \tilde{\rho}_{j}^{h} w_{j}, \\
\tilde{g}_{j}^{h k i}=\int_{0}^{1} \tilde{A}_{h k} p_{i} w_{j} d \tau, \quad \tilde{q}_{j}^{h k}=\int_{0}^{1} \tilde{A}_{h k} w_{j} d \tau, \quad \tilde{\rho}_{j}^{h}=\int_{0}^{1} \tilde{A}_{h 0} w_{j} d \tau, \\
\tilde{A}_{0}(\bar{t})=\left(T-t_{0}\right)\left[a_{0 t}\left(\left(T-t_{0}\right) \bar{t}+t_{0}\right)+\tilde{\beta}_{t}\left(\left(T-t_{0}\right) \bar{t}+t_{0}\right) Z_{t}\left(\left(T-t_{0}\right) \bar{t}+t_{0}\right)\right], \tilde{A}(\bar{t})=\left(T-t_{0}\right)\left[a_{1}-\tilde{\beta}_{t}\left(\left(T-t_{0}\right) \bar{t}+t_{0}\right) b_{1}\right]
\end{gathered}
$$




$$
\begin{gathered}
\dot{R}_{\bar{t}}=a_{1} R_{\bar{t}}+R_{\bar{t}} a_{1}^{T}-\left[R_{\bar{t}} b_{1}^{T}+\left(\tilde{c}_{10}+\sum_{r=1}^{n_{Y}+n_{X}} \tilde{c}_{1 r} \tilde{m}_{r}\right) \times \tilde{v}_{\bar{t}}\left(\tilde{c}_{20}^{T}+\sum_{r=1}^{n_{Y}+n_{X}} \tilde{c}_{2 r}^{T} \tilde{m}_{r}\right)+\sum_{r, s=1}^{n_{Y}+n_{X}} \tilde{c}_{1 r} \tilde{v}_{\bar{t}} \tilde{c}_{2 s}^{T} \tilde{K}_{r s}\right] \tilde{x}_{11}^{-1} \times \\
\times\left[R_{\bar{t}} b_{1}+\left(\tilde{c}_{20}+\sum_{r=1}^{n_{Y}+n_{X}} \tilde{c}_{2 r} \tilde{m}_{r}\right) \tilde{v}_{\bar{t}}\left(\tilde{c}_{10}^{T}+\sum_{r=1}^{n_{Y}+n_{X}} \tilde{c}_{1 r}^{T} \tilde{m}_{r}\right)+\sum_{r, s=1}^{n_{Y}+n_{X}} \tilde{c}_{2 r} \tilde{v}_{\bar{t}} \tilde{K}_{r s}\right]+\left(\tilde{c}_{10}+\sum_{r=1}^{n_{Y}+n_{X}} \tilde{c}_{1 r} \tilde{m}_{r}\right) \\
\times \tilde{v}_{\bar{t}}\left(\tilde{c}_{20}^{T}+\sum_{r=1}^{n_{Y}+n_{X}} \tilde{c}_{1 r}^{T} \tilde{m}_{r}\right)+\sum_{r, s=1}^{n_{Y}+n_{X}} \tilde{c}_{1 r} \tilde{v}_{\bar{t}} \tilde{c}_{1 s}^{T} \tilde{K}_{r s}, \\
\tilde{\beta}_{\bar{t}}=\left[R_{\bar{t}} b_{1}^{T}+\left(\tilde{c}_{10}+\sum_{r=1}^{n_{Y}+n_{X}} \tilde{c}_{1 r} \tilde{m}_{r}\right) \tilde{v}_{\bar{t}}\left(\tilde{c}_{20}^{T}+\sum_{r=1}^{n_{Y}+n_{X}} \tilde{c}_{2 r}^{T} \tilde{m}_{r}\right)+\sum_{r, s=1}^{n_{Y}+n_{X}} \tilde{c}_{1 r} \tilde{v}_{\bar{t}} \tilde{c}_{2 s}^{T} \tilde{K}_{r s}\right] \tilde{x}_{11}^{-1}, \\
\tilde{x}_{11}^{-1}=\left(\tilde{c}_{20}+\sum_{r=1}^{n_{Y}+n_{X}} \tilde{c}_{2 r} \tilde{m}_{r}\right) \tilde{v}_{\bar{t}}\left(\tilde{c}_{20}^{T}+\sum_{r=1}^{n_{Y}+n_{X}} \tilde{c}_{2 r}^{T} \tilde{m}_{r}\right)+\sum_{r, s=1}^{n_{Y}+n_{X}} \tilde{c}_{2 r} \tilde{v}_{\bar{t}} \tilde{c}_{2 s}^{T} \tilde{K}_{r s}, \\
\tilde{m}_{\bar{t}}^{\prime}=a_{0 \bar{t}}+\tilde{a}_{1} \tilde{m}_{\bar{t}}, \quad \tilde{m}(0)=m_{0},
\end{gathered}
$$$$
\tilde{K}_{\bar{t}}^{\prime}=\tilde{a} \tilde{K}_{\bar{t}}+\tilde{K}_{\bar{t}} a^{T}+\tilde{c}_{0} \tilde{v}_{\bar{t}} \tilde{c}_{0}^{T}+\sum_{r=1}^{n_{Y}+n_{X}}\left(\tilde{c}_{0} \tilde{v}_{\bar{t}} \tilde{c}_{r}^{T}+\tilde{c}_{r} \tilde{v}_{\bar{t}} \tilde{c}_{0}^{T}\right) \tilde{m}_{r}+\sum_{r, s=1}^{n_{Y}+n_{X}} \tilde{c}_{r} \tilde{\tilde{c}}_{\bar{t}} \tilde{c}_{s}^{T}\left(\tilde{m}_{r} \tilde{m}_{s}+\tilde{K}_{r s}\right), \quad K(0)=K_{0},
$$$$
\text { resolution } J \text {. }
$$$$
\tilde{a}=\bar{a}_{\bar{t}}, \quad \tilde{a}_{0}=\bar{a}_{0 \bar{t}}, \quad \tilde{c}_{r}=\bar{c}_{r \bar{t}} .
$$

Here we use wave for functions depending on dimensionless time $\bar{t}$.

Using Proposition 3 in case $a_{t}=0, b_{t}=0, b_{0 t}=0$ for Eqs (25)-(27) we get the following proposal [6].

Proposition 4. At conditions of Proposition 3 and conditions:

(iii) scalar functions $\quad \hat{\bar{X}}_{h}^{\prime}, \hat{\bar{X}}_{h}, \tilde{A}_{h k} p_{i}, \tilde{A}_{h 0}$ ( $h, k=1,2, \ldots, n_{X}$ ) belong to space $L^{2}[0,1]$;

(iv) Haar functions $w_{i}, p_{i}$ belling to space $L^{2}[0,1]$ WLLPF is defined by Eqs (36), (37).

From Proposition 4 the corresponding algorithm follow.

1) Off-line reduction of Eqs (25), (2) to dimensionless form.

2) Off-line calculation of probability moments $\tilde{m}_{\bar{t}}$ and $\tilde{K}_{\bar{t}} \quad$ of random vector $\left[\bar{Y}_{1} \ldots \bar{Y}_{n_{Y}} \bar{X}_{1} \ldots \bar{X}_{n X}\right]^{T}$ by integrating Eqs (44), (45).

3) Off-line calculation of error covariance matrix $R_{\bar{t}}$ by Eq (41) integrating.

4) Off-line calculation of parameter $\tilde{\mathfrak{x}}_{11}$ and optimal amplifier $\tilde{\beta}_{t}$ according to formulae (42) and (43).

5) Off-line introduction in space $L^{2}[0,1]$ Haar wavelet defined by Eqs (11)-(14) with maximal level of wavelet

6) Off-line calculation $p_{i}$ for Haar wavelets according to formulae (15).

7) On-line assign values $Z_{t} \quad\left(t \in\left[t_{0}, T\right]\right)$ in points $t_{j}=t_{0}+(j-1) \Delta t \quad$ for $\quad j=1,2, \ldots, L \quad ; \quad L=2 \cdot 2^{j} \quad ;$ $t \in\left[t_{0}, T\right]$.

8) On-line composition and solution SLAEq (37) for determination of coefficients $\xi_{s h}$.

9) On-line computation of error m.s. estimations $\hat{\bar{X}}_{h}$ for every component $\bar{X}_{t}$ according to formula (36).

10)On-line transition from $\bar{t} \in[1,0]$ to $t \in\left[t_{0}, T\right]$ and calculation $\hat{X}(t)$ to: $\hat{\bar{X}}(\bar{t})=\hat{X}\left(\left(T-t_{0}\right) \bar{t}+t_{0}\right)$.

\section{Suboptimal Linearized Filters at Shock Disturbances}

If the noise $V$ in Eqs (25)-(26) is autocorrelated and connected with white noise $V^{\text {sh }}$ by linear Eqs of shape filter such StSHA with parametric ShD during small sock time comparable with time of StSHA inertia are reduced to bilinear StSHA of the form [7-10]: 


$$
\begin{gathered}
\dot{X}_{t}=a_{0 t}+a_{1 t} X_{t}+a_{t} Y_{t}+\left(c_{10 t}+\sum_{r=1}^{n_{Y}} c_{1 r t} Y_{r}+\sum_{r=1}^{n_{X}} c_{1, n_{y}+r, t} X_{r}\right) N_{t}^{s h}, \quad X\left(t_{0}\right)=X_{0}, \\
\dot{Y}_{t}=b_{0 t}+b_{t} Y_{t}+b_{1 t} X_{t}+\left(c_{20 t}+\sum_{r=1}^{n_{Y}} c_{2 r t} Y_{r}+\sum_{r=1}^{n_{X}} c_{2, n_{y}+r, t} X_{r}\right) N_{t}^{s h}, \quad Y\left(t_{0}\right)=Y_{0}, \\
\dot{N}_{t}^{s h}=c_{0 t}+c_{1 t} N_{t}^{s h}+c_{t} V_{t}^{s h}, \quad N_{t}^{s h}\left(t_{0}\right)=N_{0 t}^{s h} .
\end{gathered}
$$

For given exact distribution (ED) and using equivalent linearization of bilinear functions $E_{E D} X_{h t} N_{0 t}^{s h}$ we reduce Eqs (47) to Eqs for KBF (Section 2) and for WLKBF for the composed vector $U_{t}=\left[X_{t}^{T} Y_{t}^{T} N_{t}^{s h T}\right]^{T}$ (Section 3). So we have the following Proposition.

Proposition 5. Let bilinear StSHA (47) at known exact distribution of ShD may be reduced to linear autocorrelated stochastic system with additive noises. Then KBF and WLKBF are defined by Eqs of Proposition 1 and 2 for the corresponding state vector $U_{t}$.

\section{Example}

At first let us consider KBF for the following system:

$$
\begin{gathered}
\dot{X}_{1}=X_{2}, \dot{X}_{2}=-\omega_{c}^{2} X_{1}-2 \varepsilon \omega_{c} X_{2}+S+n_{t}^{s h}+V_{1}^{s h}, \dot{X}_{3}=X_{1}, \\
Z_{1}=\dot{Y}_{1} \equiv 0, \quad Z_{2}=\dot{Y}_{2}=X_{2}+V_{2}, \quad Z_{3}=\dot{Y} \equiv 0,
\end{gathered}
$$

where $X=\left[X_{1} X_{2} X_{3}\right]^{T}$ and $Y=\left[0 Y_{2} 0\right]^{T}$ are state and observation vectors, $V_{1}^{\text {sh }}$ and $V_{2}$ are scalar independent Gaussian noises with intensities $v_{1}^{s h}$ and $v_{2}\left(v_{2} \neq 0\right)$. Note that in case of Eq (48) KBF and LPF coincide. So using Poposition 1 we have the following vector Eqs for KBF:

$$
\begin{gathered}
\dot{\hat{X}}_{t}=a_{0 t}^{s h}+\beta_{t} Z_{t}+\left(a_{1}^{s h}-\beta_{t} b_{1}\right) \hat{X}_{t}, \quad \hat{X}_{t}\left(t_{0}\right)=\hat{X}_{0} . \\
a_{0 t}^{s h}=\left[\begin{array}{c}
0 \\
S+n_{t}^{s h} \\
0
\end{array}\right], \quad a_{1}^{s h}=\left[\begin{array}{ccc}
0 & 1 & 0 \\
-\omega_{c}^{2} & -2 \varepsilon \omega_{c} & 0 \\
1 & 0 & 0
\end{array}\right], \quad b_{1}=\left[\begin{array}{lll}
0 & 0 & 0 \\
0 & 1 & 0 \\
0 & 0 & 0
\end{array}\right] . \\
\beta_{t}=R_{t} b_{1}^{T} v_{2}^{-1}=\left[\begin{array}{ccc}
0 & -\left(1 / v_{2}\right) R_{12} & 0 \\
-\left(1 / v_{2}\right) R_{22} & 0 \\
0 & -\left(1 / v_{2}\right) R_{23} & 0
\end{array}\right], \\
\dot{R}_{t}=a_{1} R_{t}+a_{1}^{T} R_{t}+v_{1}^{s h}-\beta_{t} v_{2} \beta_{t}^{T}, \quad R\left(t_{0}\right)=R_{0} .
\end{gathered}
$$

Eq (52) may be written in scalar form

$$
\left\{\begin{array}{l}
\dot{R}_{11}=2 R_{12}-\left(1 / v_{2}\right) R_{12}^{2}, \quad R_{11}\left(t_{0}\right)=R_{110} ; \\
\dot{R}_{12}=R_{22}-\omega_{c}^{2} R_{11}-2 \varepsilon \omega_{c} R_{12}-\left(1 / v_{2}\right) R_{12} R_{22}, \\
R_{12}\left(t_{0}\right)=R_{120} ; \\
\dot{R}_{13}=R_{11}+R_{23}-\left(1 / v_{2}\right) R_{12} R_{23}, \quad R_{13}\left(t_{0}\right)=R_{130} ; \\
\dot{R}_{22}=-2\left(\omega_{c}^{2} R_{12}+2 \varepsilon \omega_{c} R_{22}\right)+v_{1}^{\text {y }}-\left(1 / v_{2}\right) R_{22}^{2}, \\
R_{22}\left(t_{0}\right)=R_{220} ; \\
\dot{R}_{23}=R_{12}-\omega_{c}^{2} R_{13}-2 \varepsilon \omega_{c} R_{23}-\left(1 / v_{2}\right) R_{22} R_{23}, \\
R_{23}\left(t_{0}\right)=R_{230} ; \\
\dot{R}_{33}=2 R_{13}-\left(1 / v_{2}\right) R_{23}^{2}, \quad R_{33}\left(t_{0}\right)=R_{330} .
\end{array}\right.
$$

So we get the final Eqs for KBF:

$$
\begin{aligned}
& \dot{\hat{X}}_{1}=\left[1+\left(1 / v_{2}\right) R_{12}\right] \hat{X}_{2}-\left(1 / v_{2}\right) R_{12} Z_{2}, \\
& \dot{\hat{X}}_{3}=\hat{X}_{1}+\left(1 / v_{2}\right) R_{23} \hat{X}_{2}-\left(1 / v_{2}\right) R_{23} Z_{2}, \\
& \dot{\hat{X}}_{2}=S+n_{t}^{s h}-\omega_{c}^{2} \hat{X}_{1}-\left[2 \varepsilon \omega_{c}-\left(1 / v_{2}\right)\right] \times \\
& \times R_{22} \hat{X}_{2}-\left(1 / v_{2}\right) R_{22} Z_{2} .
\end{aligned}
$$

Secondly we use Proposition 2 and notations

$$
\dot{\hat{X}}_{t}=A_{0}+A X_{t}, \quad \hat{X}\left(t_{0}\right)=\hat{X}_{0},
$$

$$
A_{0}=\left[A_{h 0}\right]_{h}=\left[\begin{array}{c}
-\left(1 / v_{2}\right) R_{12} Z_{2} \\
-\left(1 / v_{2}\right) R_{22} Z_{2}+S+n_{t}^{s h} \\
-\left(1 / v_{2}\right) R_{23} Z_{2}
\end{array}\right],
$$

$$
\begin{gathered}
A=\left[A_{h k}\right]_{h, k}=\left[\begin{array}{ccc}
0 & 1+\left(1 / v_{2}\right) R_{12} & 0 \\
-\omega_{c}^{2} & -\left[2 \varepsilon \omega_{c}-\left(1 / v_{2}\right)\right] R_{22} & 0 \\
1 & 0 & \left(1 / v_{2}\right) R_{23}
\end{array}\right], \\
\hat{\bar{X}}_{t}^{\prime}=\bar{A}_{0}+\overline{\bar{A}} \hat{\bar{X}}, \quad \hat{\bar{X}}(0)=\hat{X}_{0},
\end{gathered}
$$

$$
\bar{A}_{0}=\left[\bar{A}_{h 0}\right]_{h}=\left(T-t_{0}\right)\left[\begin{array}{c}
-\left(1 / v_{2}\right) R_{12} Z_{2} \\
-\left(1 / v_{2}\right) R_{22} Z_{2}+S+n_{t}^{s h} \\
-\left(1 / v_{2}\right) R_{23} Z_{2}
\end{array}\right] \text {, }
$$


$\bar{A}=\left[\bar{A}_{h k}\right]_{h, k}=\left(T-t_{0}\right)\left[\begin{array}{ccc}0 & 1+\left(1 / v_{2}\right) R_{12} & 0 \\ -\omega_{c}^{2} & -\left[2 \varepsilon \omega_{c}-\left(1 / v_{2}\right)\right] R_{22} & 0 \\ 1 & 0 & \left(1 / v_{2}\right) R_{23}\end{array}\right]$.

So we get the final WLKBF Eqs:

$$
\begin{aligned}
& {\left[\bar{R}_{11}^{\prime}=\left(T-t_{0}\right)\left[2 R_{12}-\left(1 / v_{2}\right) R_{12}^{2}\right], \quad \bar{R}_{11}\left(t_{0}\right)=R_{110} ;\right.} \\
& \bar{R}_{12}^{\prime}=\left(T-t_{0}\right)\left[R_{22}-\omega_{c}^{2} R_{11}-2 \varepsilon \omega_{c} R_{12}-\right. \\
& \left.-\left(1 / v_{2}\right) R_{12} R_{22}\right], \quad \bar{R}_{12}\left(t_{0}\right)=R_{120} \text { ； } \\
& \bar{R}_{13}^{\prime}=\left(T-t_{0}\right)\left[R_{11}+R_{23}-\left(1 / v_{2}\right) R_{12} R_{23}\right] \text {, } \\
& \bar{R}_{13}\left(t_{0}\right)=R_{130} \text {; } \\
& \left\{\bar{R}_{22}^{\prime}=\left(T-t_{0}\right)\left[v_{t}^{s h}-2\left(\omega_{c}^{2} R_{12}+2 \varepsilon \omega_{c} R_{22}\right)-\right.\right. \\
& \left.-\left(1 / v_{2}\right) R_{22}^{2}\right], \quad \bar{R}_{22}\left(t_{0}\right)=R_{220} \text {; } \\
& \bar{R}_{23}^{\prime}=\left(T-t_{0}\right)\left[R_{12}-\omega_{c}^{2} R_{13}-2 \varepsilon \omega_{c} R_{23}-\right. \\
& \left.-\left(1 / v_{2}\right) R_{22} R_{23}\right], \quad \bar{R}_{23}\left(t_{0}\right)=R_{230} \text {; } \\
& \bar{R}_{33}^{\prime}=\left(T-t_{0}\right)\left[2 R_{13}-\left(1 / v_{2}\right) R_{23}^{2}\right] \text {, } \\
& \bar{R}_{33}\left(t_{0}\right)=R_{330} \text {. } \\
& \hat{\bar{X}}_{h}=\sum_{s=1}^{L} c_{h s} p_{s} \bar{X}_{0 h}, \\
& c_{h s}=\sum_{k=1}^{3} \sum_{i=1}^{L} c_{h i} g_{s}^{h k i}+\hat{X}_{o h} \sum_{i=1}^{L} q_{s}^{h k}+\rho_{s}^{h} \\
& (k=1,2, \ldots, p, \quad s=1,2, \ldots, L),
\end{aligned}
$$

$g_{j}^{h k i}=\int_{0}^{1} \bar{A}_{h k}(\tau) p_{i}(\tau) w_{s}(\tau) d \tau$,

$q_{j}^{h k}=\int_{0}^{1} \bar{A}_{h k}(\tau) w_{s}(\tau) d \tau, \quad \rho_{j}^{h}=\int_{0}^{1} \bar{A}_{h 0}(\tau) w_{s}(\tau) d \tau$.

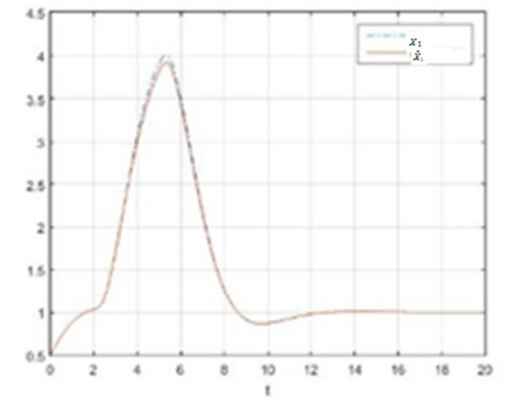

(a)
Computer experiments "Figures 1-15" were realized for the following values of parameters: $\omega_{c}=1 ; \quad S=1 ; \quad v_{2}=1, \quad j=5$ and $\varepsilon=0,7 ; \varepsilon=0,1$. The following variants of $\mathrm{ShD}$ were considered.

1) Deterministic $\mathrm{ShD}$ with $n_{t}^{s h}$ and stochastic $\mathrm{ShD}$ with $v_{t}^{s h}$ at $t_{s h}=2,1875, \Delta n=3, \Delta t=3$.

$$
\begin{aligned}
& n_{t}^{s h}=\left\{\begin{array}{ccc}
\Delta n, & \text { at } & t \in\left[t_{s h} ; t_{s h}+\Delta t\right], \\
0, & \text { at } & t \notin\left[t_{s h} ; t_{s h}+\Delta t\right],
\end{array}\right. \\
& v_{t}^{s h}=\left\{\begin{array}{ccc}
\Delta n, & \text { at } & t \in\left[t_{s h} ; t_{s h}+\Delta t\right], \\
0, & \text { at } & t \notin\left[t_{s h} ; t_{s h}+\Delta t\right] .
\end{array}\right.
\end{aligned}
$$

2) Deterministic $\mathrm{ShD} n_{t}^{s h}$ and stochastic $\mathrm{ShD} v_{t}^{s h}$ at $t_{\text {sh }}=2,1875$

$$
\begin{aligned}
& n_{t}^{s h}=\left\{\begin{array}{lll}
10, & \text { at } & t \in\left[t_{s h} ; t_{s h}+5\right], \\
0, & \text { at } & t \notin\left[t_{s h} ; t_{s h}+5\right],
\end{array}\right. \\
& v_{t}^{s h}=\left\{\begin{array}{lll}
6, & \text { at } & t \in\left[t_{s h} ; t_{s h}+7\right], \\
0, & \text { at } & t \notin\left[t_{s h} ; t_{s h}+7\right] .
\end{array}\right.
\end{aligned}
$$

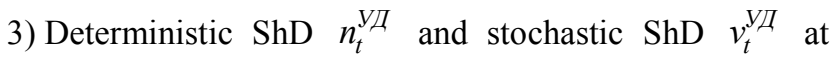
$t_{\text {уд }}=0,135$,

$$
\begin{aligned}
& n_{t}^{s h}=\left\{\begin{array}{lll}
10, & \text { at } & t \in\left[t_{s h} ; t_{s h}+1\right], \\
0, & \text { at } & t \notin\left[t_{s h} ; t_{s h}+1\right],
\end{array}\right. \\
& v_{t}^{s h}=\left\{\begin{array}{lll}
6, & \text { at } & t \in\left[t_{s h} ; t_{s h}+1\right], \\
0, & \text { at } & t \notin\left[t_{s h} ; t_{s h}+1\right] .
\end{array}\right.
\end{aligned}
$$

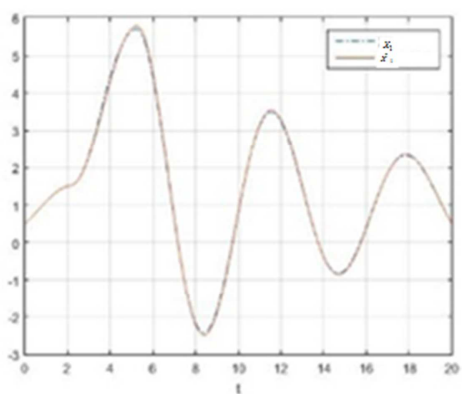

(b)

Figure 1. Plot realization of $X_{1}$ and its estimation (variant 1) for $\varepsilon=0,7$ (a) and $\varepsilon=0,1$ (b).

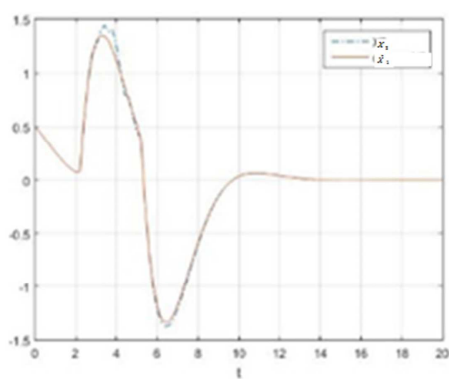

(a)

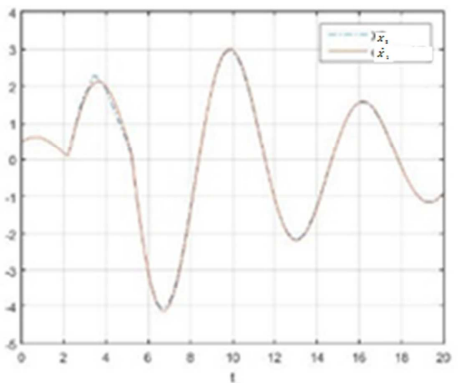

(b)

Figure 2. Plot realization of $X_{2}$ and its estimation (variant 1) for $\varepsilon=0,7$ (a) and $\varepsilon=0,1$ (b). 


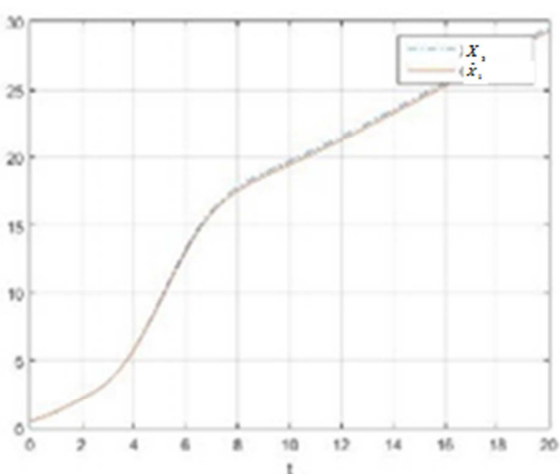

(a)

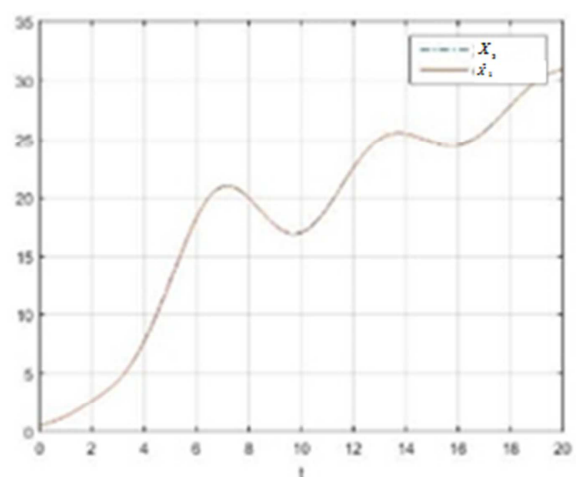

(b)

Figure 3. Plot realization of $X_{3}$ and its estimation (variant 1) for $\varepsilon=0,7$ (a) and $\varepsilon=0,1$ (b).

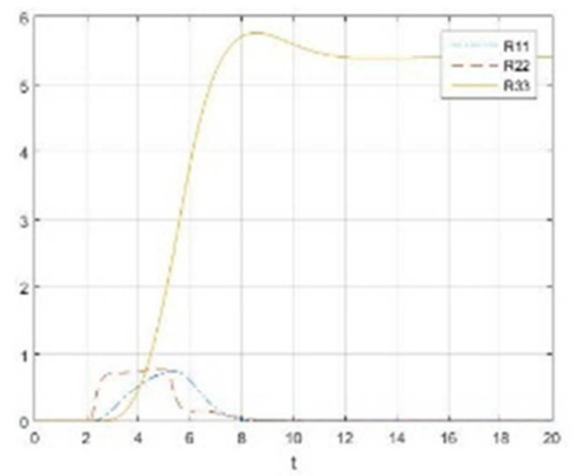

(a)

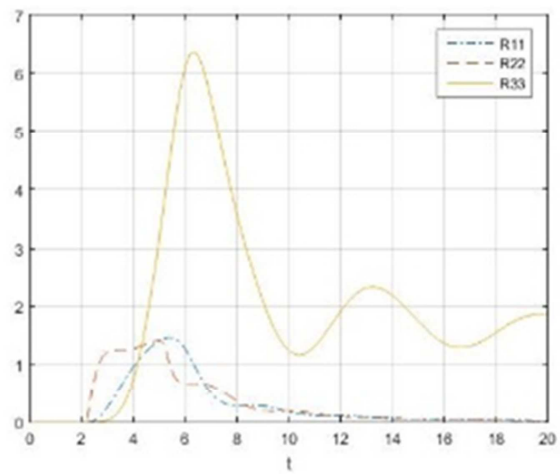

(b)

Figure 4. Filter error variance plots $R_{11}, R_{22}, R_{33}$ (variant 1) for $\varepsilon=0,7$ (a) and $\varepsilon=0,1$ (b).

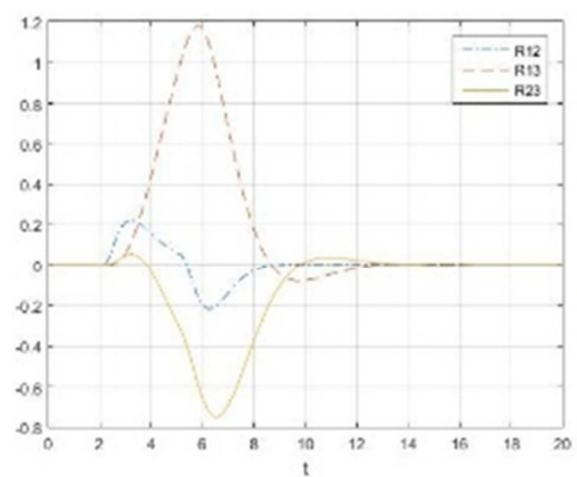

(a)

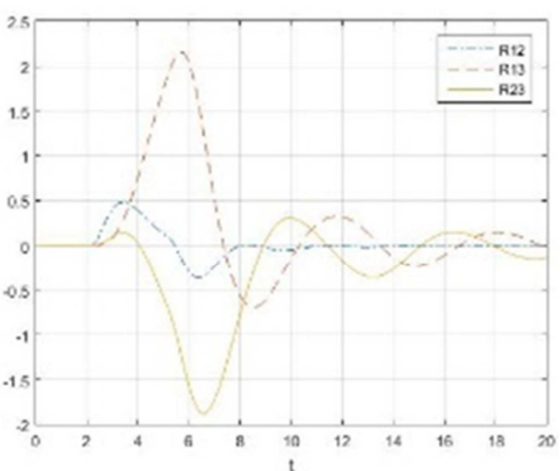

(b)

Figure 5. Filter error variance plots $R_{12}, R_{13}, R_{23}$ (variant 1) for $\varepsilon=0,7$ (a) and $\varepsilon=0,1$ (b).

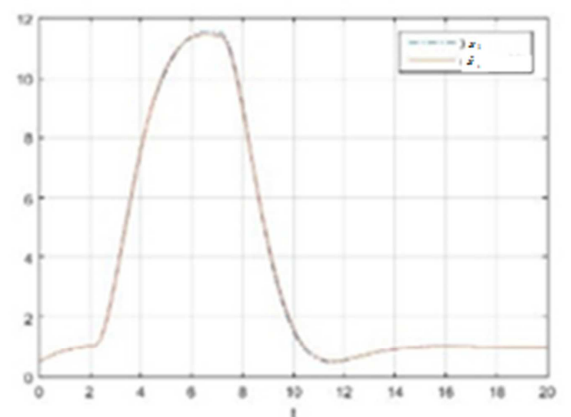

(a)

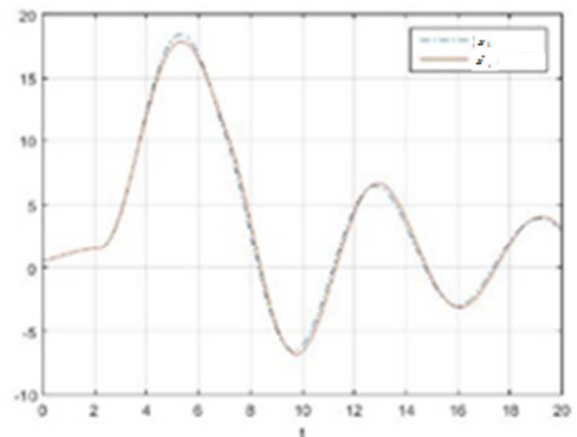

(b)

Figure 6. Plot realization of $X_{1}$ and its estimation (variant 2) for $\varepsilon=0,7$ (a) and $\varepsilon=0,1$ (b). 


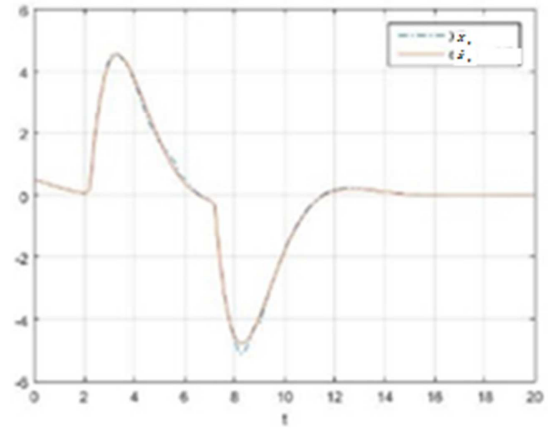

(a)

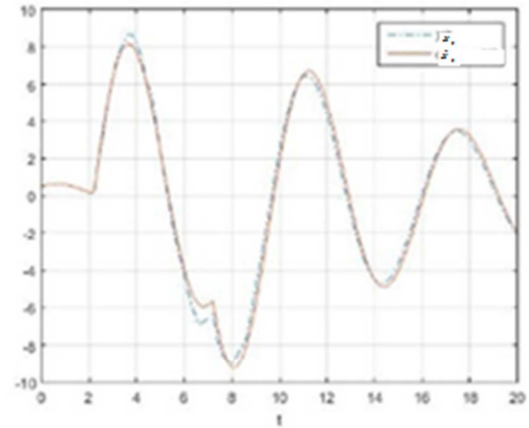

(b)

Figure 7. Plot realization of $X_{2}$ and its estimation (variant 2) for $\varepsilon=0,7$ (a) and $\varepsilon=0,1$ (b).

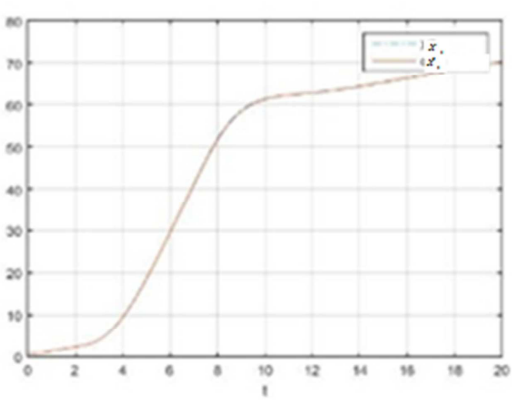

(a)

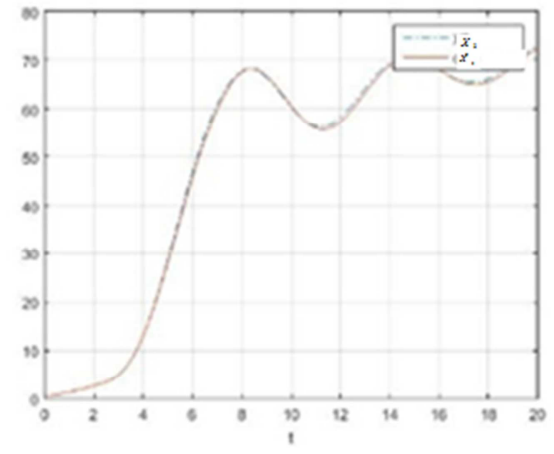

(b)

Figure 8. Plot realization of $X_{3}$ and its estimation (variant 2) for $\varepsilon=0,7$ (a) and $\varepsilon=0,1$ (b).

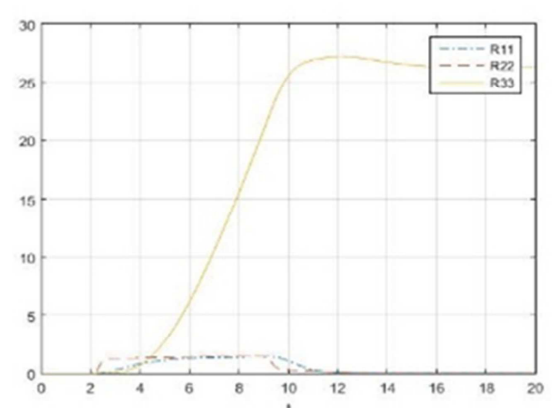

(a)

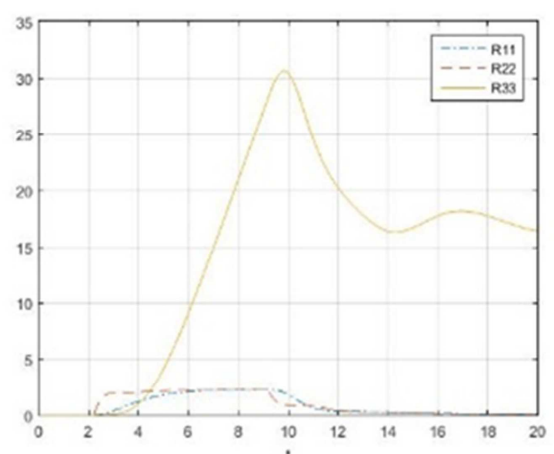

(b)

Figure 9. Filter error variance plots $R_{11}, R_{22}, R_{33} \quad$ (variant 2) for $\varepsilon=0,7$ (a) and $\varepsilon=0,1$ (b).

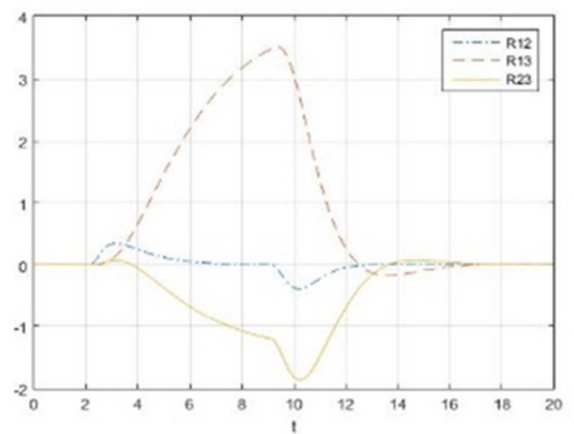

(a)

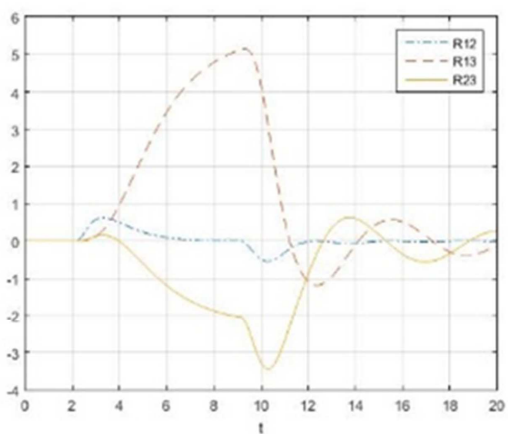

(b)

Figure 10. Filter error variance plots $R_{12}, R_{13}, R_{23}$ (variant 2) for $\varepsilon=0,7$ (a) and $\varepsilon=0,1$ (b). 


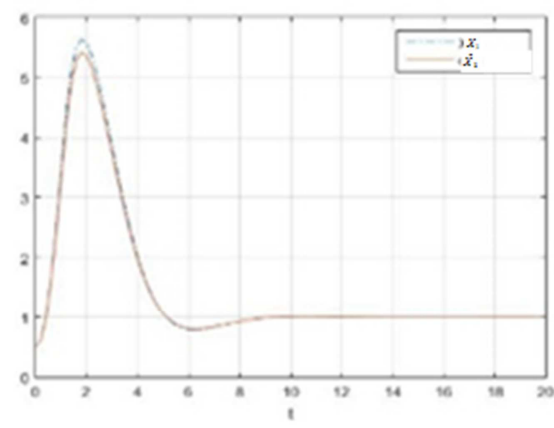

(a)

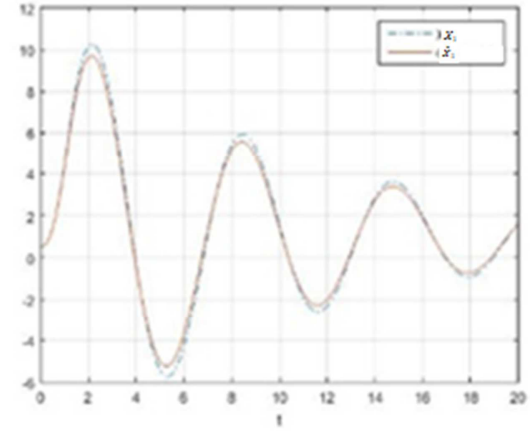

(b)

Figure 11. Plot realization of $X_{1}$ and its estimation (variant 3) for $\varepsilon=0,7$ (a) and $\varepsilon=0,1$ (b).

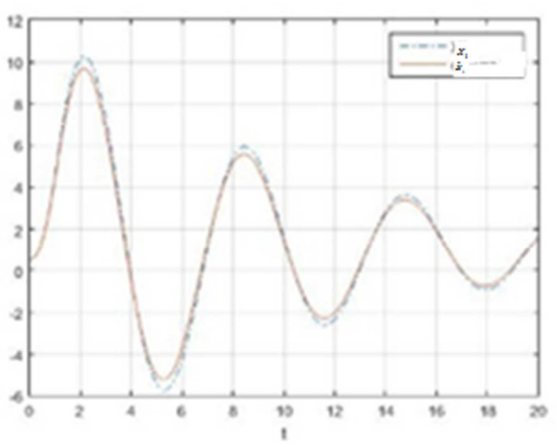

(a)

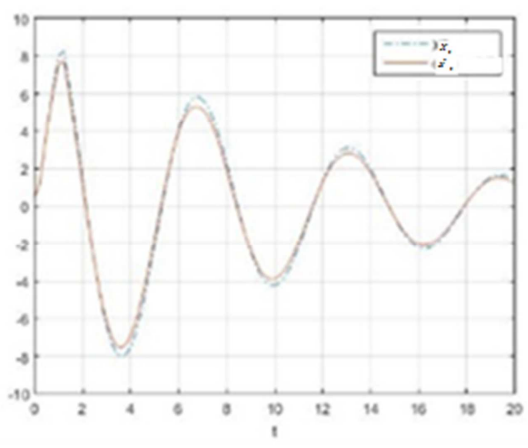

(b)

Figure 12. Plot realization of $X_{2}$ and its estimation (variant 3) for $\varepsilon=0,7$ (a) and $\varepsilon=0,1$ (b).

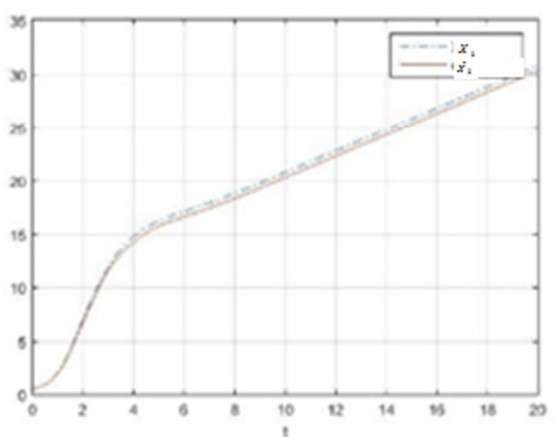

(a)

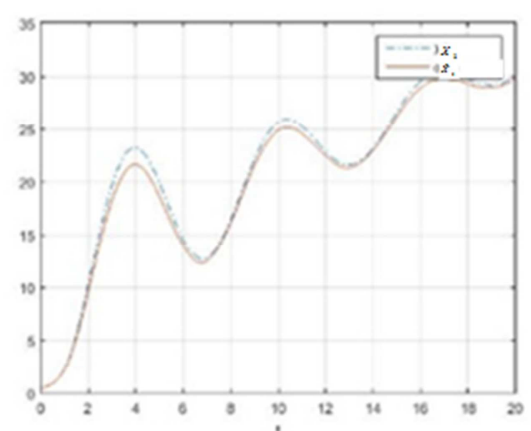

(b)

Figure 13. Plot realization of $X_{3}$ and its estimation (variant 3) for $\varepsilon=0,7$ (a) and $\varepsilon=0,1$ (b).

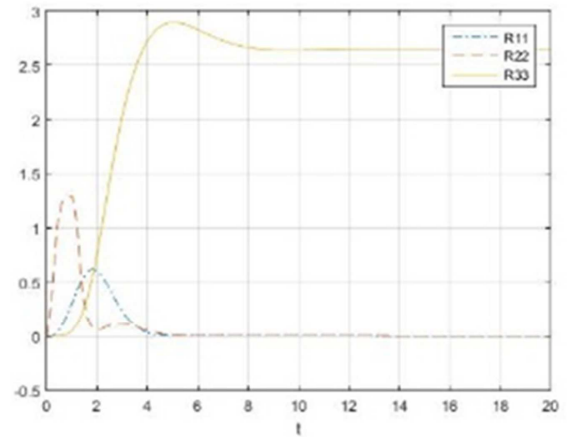

(a)

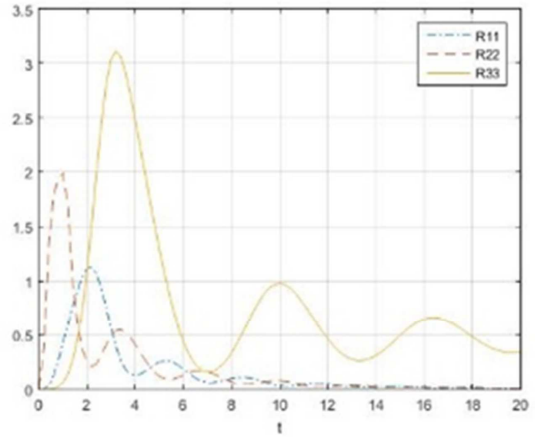

(b)

Figure 14. Filter error variance plots $R_{11}, R_{22}, R_{33}$ (variant 3) for $\varepsilon=0,7$ (a) and $\varepsilon=0,1(b)$. 


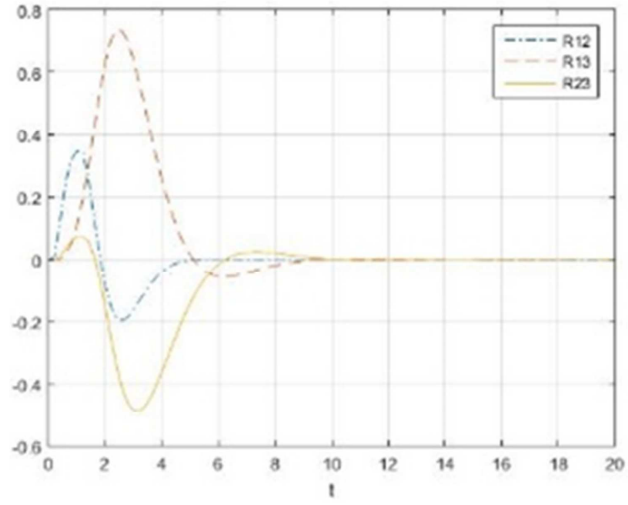

(a)

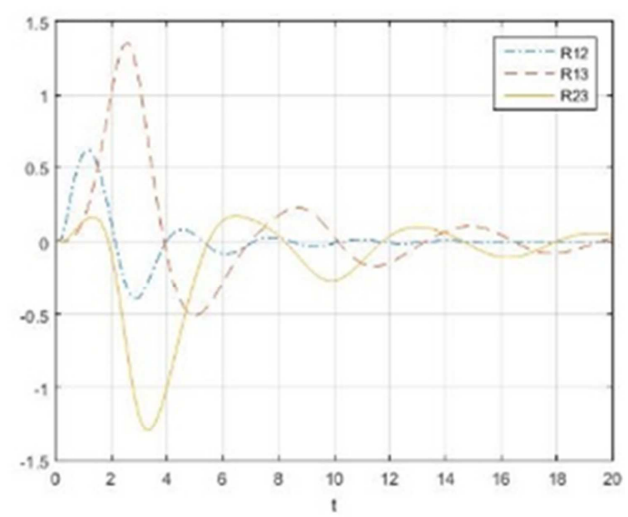

(b)

Figure 15. Filter error variance plots $R_{12}, R_{13}, R_{23}$ (variant 3) for $\varepsilon=0,7$ (a) and $\varepsilon=0,1$ (b).

\section{Conclusion}

For filtering problems in StSHA under nonGaussian ShD methodological and algorithmically WL support is developed. 3 types of filters are considered: KBF (WLKBF), LPF (WLLPF) and SOLF (WLSOLF). These filters have the following advantages: on-line regime, high accuracy and possibility of algorithmically description of complex ShD. Wavelet filter modifications are based Galerkin method and Haar wavelet expansions. WLF unlike KBF, LPF and SOLF do not need to integrate system of ordinary differential Eqs. These filters must solve system of linear algebraic Eqs with constant coefficients.

KBF (WLKBF) and SOLF (WLSOLF) are recommended for StSHA with additive ShD whereas LPF (WLLPF) are recommended for StSHA with parametric and additive ShD.

Basic applications are on-line identification and calibration of nonstationary processes in StSHA at ShD.

Methods are illustrated by example of 3 dimensional differential linear information control system at complex ShD. Basic algorithms and error analysis for KBF (WLKBF) and LPF (WLLPF) are presented on 15 figures illustrate filters popularities for small and big damping. These filters allow to estimate the accumulation effects for systematic and random errors.

Results may be generalized for filtration, extrapolation and interpolation problems in StSHA with multiple ShD.

\section{Acknowledgements}

The authors thanks Federal Research Center "Computer Sciences and Control" for reporting the work presented in this article. Authors much obliged to Mrs Irina Sinitsyna and Mrs Helen Fedotova for translation and manuscript preparation.

\section{References}

[1] Sinitsyn I. N., Sergeev I. V., Korepanov E. R., Konashenkova T. D. Software tools for analysis and synthesis of stochastic systems with high availability (IV). Highly Available Systems. 2017. V. 13. № 3. P. 55-69.
[2] Sinitsyn I. N., Sergeev I. V., Korepanov E. R., Konashenkova T. D. Software tools for analysis and synthesis of stochastic systems with high availability (V). Highly Available Systems. 2018. V. 14. № 1. P. 59-70.

[3] Sinitsyn I. N., Sergeev I. V., Korepanov E. R., Konashenkova T. D. Software tools for analysis and synthesis of stochastic systems with high availability (VI). Highly Available Systems. 2017. V. 14. № 2. P. 40-56.

[4] Sinitsyn I. N., Zykov D. V., Korepanov E. R., Konashenkova T. D. Software tools for analysis and synthesis of stochastic systems with high availability (VII). Highly Available Systems. 2019. V. 15. № 1. P. 47-61.

[5] Sinitsyn I. N., Zykov D. V., Korepanov E. R., Konashenkova T. D. Software tools for analysis and synthesis of stochastic systems with high availability (VIII). Highly Available Systems. 2019. V. 15. № 1. P. 62-69.

[6] Sinitsyn I. N., Sinitsyn V. I., Korepanov E. R., Konashenkova T. D. Software tools for analysis and synthesis of stochastic systems with high availability (X). Highly Available Systems. 2020 . V. 16. № 4. P. 24-39. DOI: 10.18127/j20729472-202004-02 (In Russian).

[7] Pugachev V. S., Sinitsyn I. N. Stochastic Differential Systems. Analysis and Filtering, John Wiley \& Sons, Chichester, 1987.

[8] Pugachev V. S., Sinitsyn I. N. Stochastic Systems. Theory and Applications, World Scientific, Singapore, 2001.

[9] Socha L. Linearization Methods for Stochastic Dynamic Systems. Lect. Notes Phys. 730. Springer, Berlin Heidelberg, 2008.

[10] Sinitsyn I. N. Kalman and Pugachev Filtering, Torus Press, Moscow, 2005, $1^{\text {st }}$ ed; 2007, $2^{\text {nd }} e d$.

[11] Heil C., Walnut D. F. Fundamental Papers om Wavelet Theory. Princeton University Press, Princeton, New-Jersey, 2006.

[12] Percival D. B., Walden A. T. Wavelet Methods for Time Series Analysis. Cambridge University Press, Cambridge, 2000.

[13] Gagnon L., Lina J. M. (1994). Symmetric Daubechies' wavelets and numerical solutions of NLS2 equations. J. Phys. A: Math. Gen. 27: 8207-8230.

[14] Xu J., Shann W. (1992). Galerkin-wavelet methods for two point value problems. Number. Math. 63: 123-144. 
[15] Lepik U. (2005). Numerical solution of differential equations using Haar wavelets. Mathematics and Computers in Simulation, 68: 127-143.
[16] Nason G. P. Wavelet Methods in Statistics with R, 2008, Springer Science Business Media, LLC. 